\title{
Knowledge-Based Seismogram Processing by Mental Images
}

\author{
Manfred Joswig, Member, IEEE
}

\begin{abstract}
The impact of pictorial knowledge representation is demonstrated for two examples of time series analysis in seismology. The approaches perform a) automated recognition of known event signatures and b) high-resolution onset timing of later phases. Both methods work well under extreme conditions of noise and achieved human-like performance in recognizing known situations. Crucial for this success of pictorial knowledge representation was the design of suitably scaled images. They must be simple and robust enough to transform the complexity of "real life" data into a limited set of patterns. These patterns differ significantly from the initial data; they correspond more closely to the non-linear weighting of recognized impressions by an experienced scientist. Thus we address the pictorial presentations as mental images.

For both reported applications, part of their power comes by model-based image modifications. However, this enhancement is far from demanding a complete theory. Any fractional model already enhances the image adaptation, so mental images are best suited to deal with incomplete knowledge like any other artificial intelligence approach.

Cognitive plausibility was found for both the non-linear image scalings and the model-based image modifications. In general, our way of pictorial knowledge representation conforms to the concept of mental images by Kosslyn. Any new task will demand the composition of new, dedicated image transformations where some generalized design criteria are derived from our applications.
\end{abstract}

\section{INTRODUCTION}

$\mathbf{T}$ HE INSTRUMENTAL observation of earthquake ground motion by seismogram registration began at the end of last century when a strong Japanese earthquake was recorded and correctly identified in Potsdam, Germany. A hundred years later seismic observatories all over the world monitor these prominent events. Additionally, they get some 10000 weaker earthquakes per year by the improvements to high-resolution array techniques and reliable digital analysis methods which lowered the monitoring threshold down to the level of permanent ground noise. The distances between epicenter and recording station range from local $(<1000 \mathrm{~km})$ and regional $(<3000 \mathrm{~km})$ to teleseismic. This distinction is by no means arbitrary; instead it mirrors the differences in wave propagation for the principal layers of planet earth, namely crust, mantle, and core. The variety of seismogram signatures gets most extreme for teleseismic where additional effects like focusing of body waves or differences in surface wave propagation for

Manuscript received June 8, 1991; revised April 6, 1993. This work was supported by Deutsche Forschungsgemeinschaft under grant Ha 842/8-1.

The author was with the Institut für Geophysik, Ruhr-Universität Bochum, Germany. He is now with Lehrstuhl für Meßtechnik, RWTH Aachen, D-52056 Aachen, Germany.

IEEE Log Number 9214598. continental and oceanic crust occur. They will significantly change the amplitude ratios and dispersion characteristics even for one earthquake and adjacent stations. Then add different hypocenter depths, different source mechanisms of, e.g., shear fault or explosion, some medium anisotropy plus noise interferences, and you get the complexity of "real life" data. For the interested reader, an introduction can be found in textbooks of Bolt [1], [2].

The approaches for earthquake processing reflect this complexity. They range from positions like each earthquake is individual to the attempts of master event correlation for repetitive sources. Still in common, they have the merely indirect access to the scientifically interesting phenomena. This access is based on seismogram interpretation and performed by at least four steps of routine processing, namely (I) detection, (II) phase association, (III) location and (IV) source identification (e.g., discrimination between earthquake or nuclear explosion for disarmament control).

All of these four steps can also be performed automatically, either by procedural approaches or knowledge-based which better copes with our restricted experience and limited theoretical modeling. Both stages (III) and (IV) can rely on explicit rules to exploit the concepts of symbolic knowledge representation by frames, scripts or semantic nets. So the reported solutions basically describe expert systems [3]-[6]. Instead, in the scope of this paper we will focus on aspects of the first two tasks, namely earthquake detection and phase association. Here the need of automatization is even more urgent since both stages run on the permanent stream of real-time data and must convert the subsymbolic information contained in the seismograms into entities like seismic event or phase onset time to start up the rule-based reasoning. In addition to the earthquakes, all the registrations are contaminated by a multitude of noise events with often higher amplitude which put heavy load on every observatory work. These noise events are caused by civilization (explosions, traffic noise, industry reverberations, and sonic bangs) or natural sources (wind gusts and rise of ocean breakers). Any automated seismogram processing must cope with these disturbances and try to suppress them as early as possible. So the decisive criterion for a good approach is its low false alarm rate paired with high detection probability.

The first efforts in automated earthquake processing date back to the 1960 's and focus on the detection task; the proposed methods are based on optimum filters, amplitude statistics and decision theory (for a comparative overview 
see [7]). They all share one inherent assumption: Given the stationary level of ground noise, any temporary deviation is a possible candidate for an earthquake. So all noise pulses must be taken, too, and any sufficient detection sensitivity results in a high single-station false alarm rate. Only the postprocessing by coincidence criteria upgraded the detection performance of a whole network of stations to some acceptable limits and let to practical implementations [8].

\section{Mental Images and Pattern Recognition}

The alternative to the previous, filter-based approaches is to test on the positive agreement in signature once the desired earthquakes or unwanted noise bursts have been characterized. This scheme equals more closely the human inspection of seismogram traces-the experienced seismologist can discriminate a whole ensemble of common signals at first glance. As obvious as this application was for pattern recognition, as clear was its initial failure. The variety of seismogram signatures is sheer unpredicable; the necessary generalization of waveforms performed by propositional descriptions in syntactic entities [9] or grammars [10] did not succeed in routine works.

\section{A. Analogue Knowledge Representation}

In the scope of this paper, we introduce some earthquake recognition schemes that alternatively rely on analogue knowledge representation. Approaches of this kind were discussed in the Imagery Debate [11]-[13]. As one conclusion, pictorial knowledge representations promise to be especially suited for acquisition and application problems ("knowing how") like the earthquake recognition while knowledge justification ("knowing that") is dominated by theses and clauses corresponding to expert systems [14].

Utilizing the analogue approach by computer will result in modeling human vision which is admittedly extremely difficult because of the many mental processes that are performed unconsciously. However, the situation gets different when we change from everyday life to scientific applications as in seismology. Every conducted experiment can be seen as an improvement of the scientist's natural senses. Human vision is the primary interface to recognize these new, artificial sensors; the relevant images, however, are mental images of experience and not yet pictures on computer screens, paper maps, or optical objectives. When the scientist proceeds from the initial, fuzzy vision and an uncoordinated, babylike touch of his experimental setups to the purposeful view and precise manipulation to get the admired "expert," this is a conscious process of an adult person experienced and endured within some few years. So this learn to see is at least partly controllable by introspective and not descended from evolution-only our truly experienced and matured colleagues can't explain their path of viewing anymore. In the following chapters, we will focus on the very idea of mental images, so all descriptions are done by images as well. The details of computational implementations, the achieved results, some tradeoff comparison to related approaches, and our recommendations of the most suited algorithm for different situations
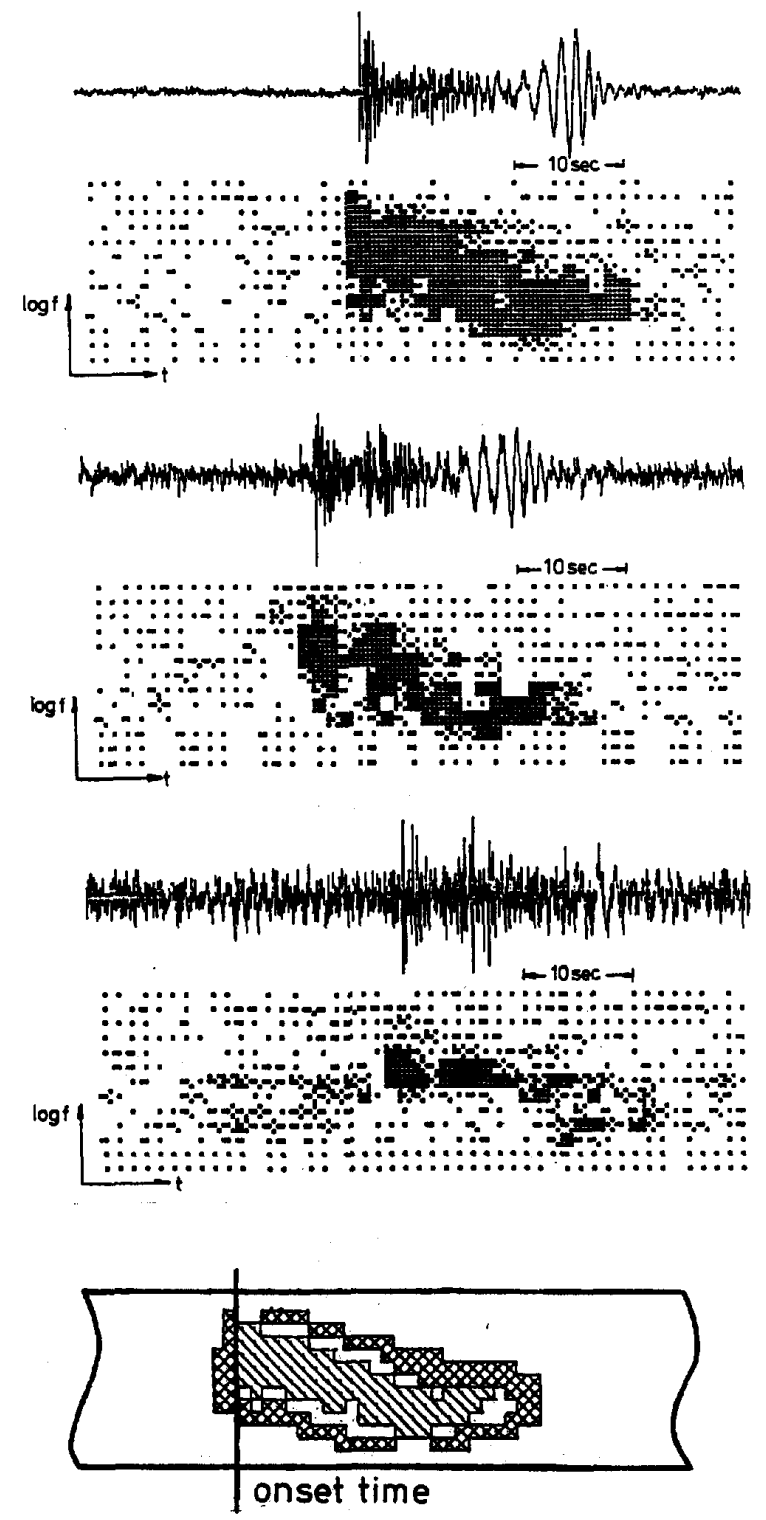

Fig. 1. For the automated earthquake recognition, the mental images correspond best to the information content of sonograms which represent the short-term spectral energy above a time-frequency plane. All the seismograms (i.e., time series) in this figure are caused by local earthquakes from one epicenter cluster in 40-km distance to the recording station. The corresponding sonograms are shown; they are scaled according to our initial, intuitive attempts where blackness codes intensity. The pattern recognition is performed by horizontal shift of contour matchers which make up our pictorial knowledge base. Only the one appropriate pattern for the epicenter cluster of this example is shown in the last row. Its striped areas demand signal energy (i.e., blackness), the crossed border performs edge conversion by test on no energy.

can be found elsewhere in the seismological literature [7], [15]-[18]. Instead, in this paper we care about behavioral details of human seismogram processing that are usually not mentioned in textbooks but do compress the gained experience of routine works. 

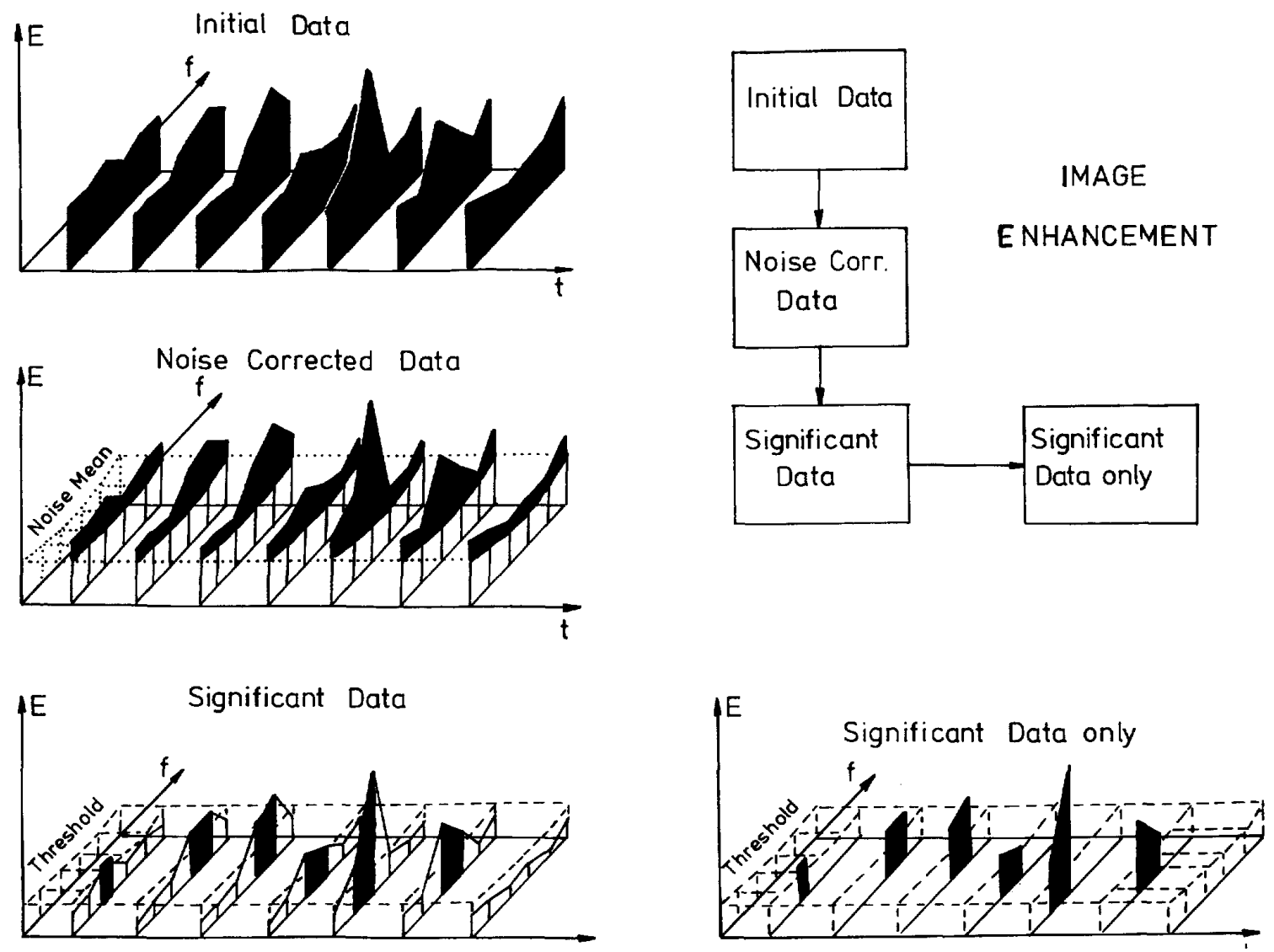

Fig. 2. The best possible image enhancement for signal detection is based on a physical model. It takes the seismic ground noise per frequency band as statistically constant at least for the duration of the earthquake. So we can completely describe the noise by mean (dotted line) and variance. First we correct all short-term spectral energy by the offset of noise mean. Then only those data remain significant which are above some threshold (the dashed line, commonly one times variance).

\section{B. Pattern Recognition on Mental Images}

As a first attempt, our approach for earthquake recognition was restricted to the single-trace registration of local seismicity and was based on a simple observation: Seismologists focus their attention on shifts of spectral energy instead of maximum amplitudes while scanning through the sheer amount of 24 hours of seismogram plotting. They utilize the theoretically confirmed experience that earthquake signals are made up by different phases which propagate by different velocities with distinct dominant frequencies. Noise bursts, on the other hand, can be characterized by monochrome wave trains or isolated pulses. So a representation of spectral energy versus time could in principle resolve this difference by image. These PSD plots are common place in seismology and known as sonograms or spectrograms; they were, however, never used for automated processing. In the application of earthquake detection, it was initially understood that sonograms can only be used if they are stable against the minor changes in event signature. Then we can reduce the manifold of individual seismograms to a manageable subset of event types that can be recognized by some distinct patterns.
Our goal of robustness was reached by drastic reductions in dynamic range and pixel resolution performed by highly non-linear transformations on the initial sonograms. A detailed description of the whole algorithm is given in [7]; the result to mention here is that the sonograms of Fig. 1 contain 1000 times less information than the corresponding seismogram plots shown above. The 3 seismic events are examples for the same type of earthquake but each with different magnitude. Despite this variation, the calculated sonograms are so robust that they can be recognized by one pattern. It performs as a contour matcher with edge inversion on a sliding black/white threshold. By the recognition of pattern types, the overall detector system converts the subsymbolic information of time series into symbolic entities that will serve as basic assumptions for the further reasoning.

The scaling and recognition procedures were selected intuitively but not arbitrarily. They should follow the cognitive plausibility of our self-observations. For local earthquake data, seismologists will distinguish the frequency shift from $1 \mathrm{~Hz}$ to $2 \mathrm{~Hz}$ very reliably but tend to ignore the same difference between $11 \mathrm{~Hz}$ and $12 \mathrm{~Hz}$. The subjective impressions in 

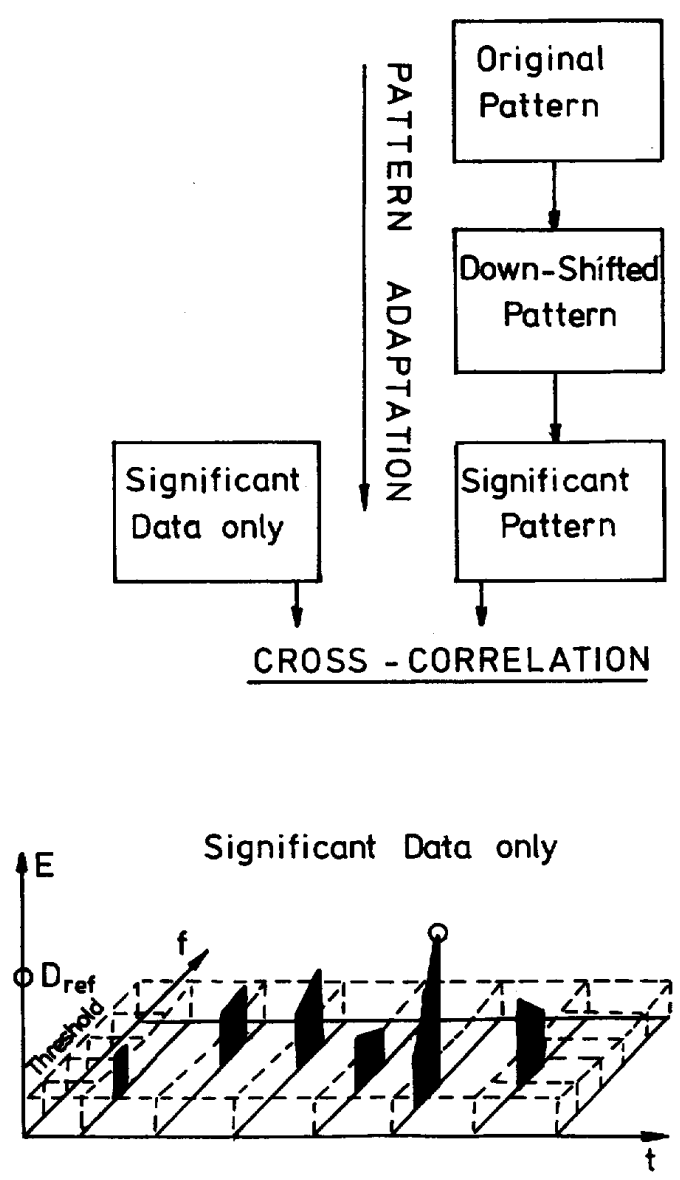
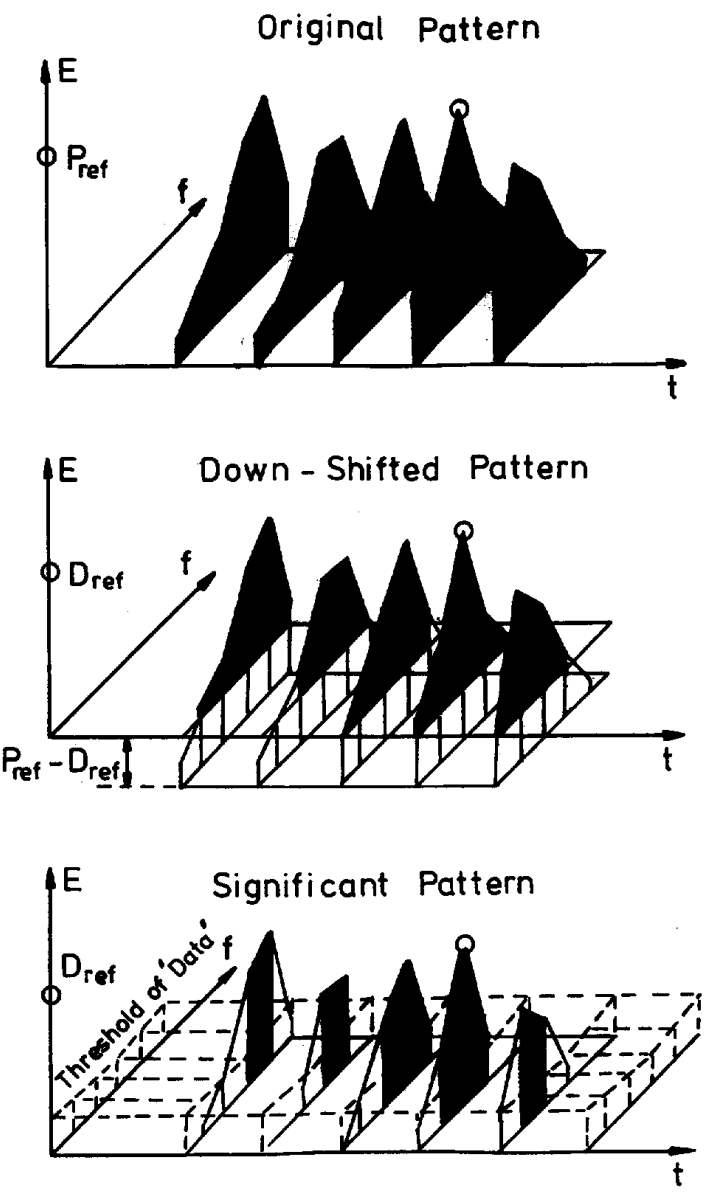

(a)

Fig. 3. The qualitative idea of pattern adaptation is explained for an idealized pattern without noise that is compared to the significant signal energy of Fig. 2. Now the inherent physical model describes how the intensity mountains of the known pattern will be masked by the highlands of stationary ground noise once we adapt the pattern amplitude at some reference sample (the circled peak) to the corresponding sample of actual data. As a result of this downshift, many details of the pattern get undetectable; thus they must be taken as significant for no energy in the subsequent pattern recognition. When in (b) the data have proceeded one image sample, the pattern is adapted once again. This time, the remaining fractions is so small (only 5 from 29 values) that pattern recognition, i.e. calculating the cross-correlation, will be skipped.

amplitude variation also follow non-linear weighting. The seismologist tests on existence of signal energy and the predictable absence at other times; this is modelled in image recognition by the edge inversion. In our course of image design, the ideas of von Neumann [19] supported the robust, coarse grain simplifications that resulted in 13 frequency bands, 47 time samples and 9 amplitude steps for the sonograms in Fig. 1.

In general, sonograms are just arbitrary mappings of spectral energy over time. The suitably scaled sonograms used here, however, were already understood as a possible visualization of internal or mental images corresponding to the impressions of subjective significance. The patterns for automated recognition had to conform to these mental images and not to the pictures of visible data. Other characteristics of mental images remained unclear and a complete definition was still out of range.

\section{Mental Images AND Mental Modifications}

The success of the initial sonogram-detector encouraged the way of pictorial knowledge representation; however, some problems remained unsolved. Even for similar event signatures in seismic arrays, the set of patterns had to be tuned individually for each station to reflect the differences in ambient ground noise; the sonogram scaling was robust only within some limited dynamic range and not as sensitive as possible. The seismologist, in contrast, is able to avoid these shortcomings by some reasoning. Of course, he also can't predict the actual energy distribution of earthquake signals or the course of seismic ground noise but he can rely on fragments of theory that exist for the statistical behavior and the interaction of both processes. In routine inspection, these ideas transform to some mental image processing that guide the seismologist's 


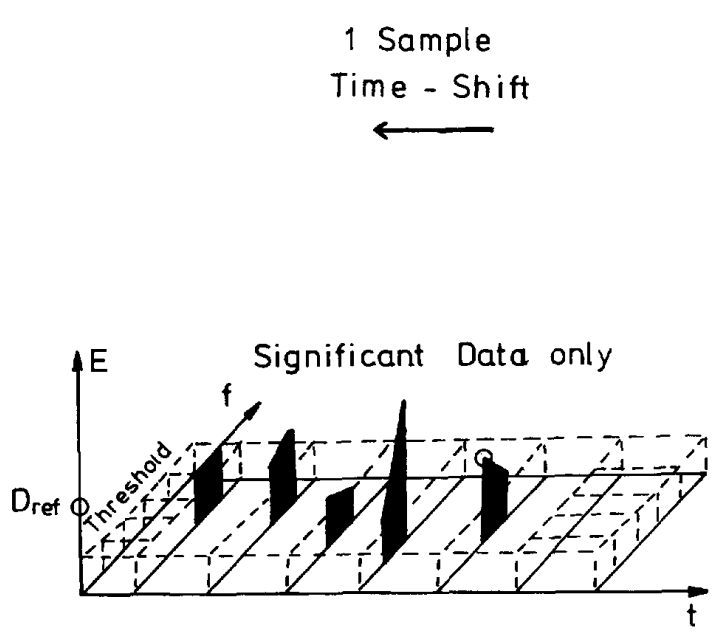

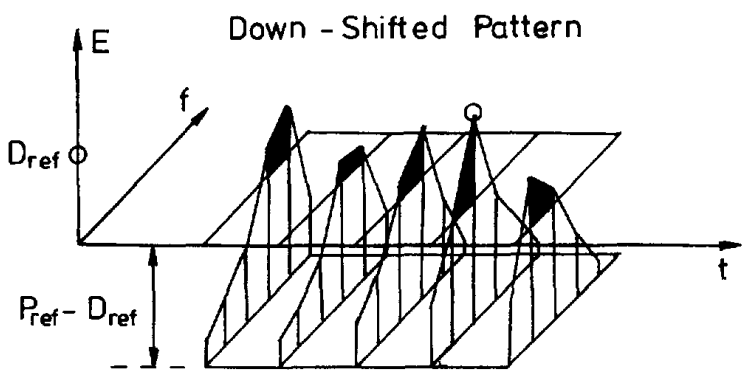

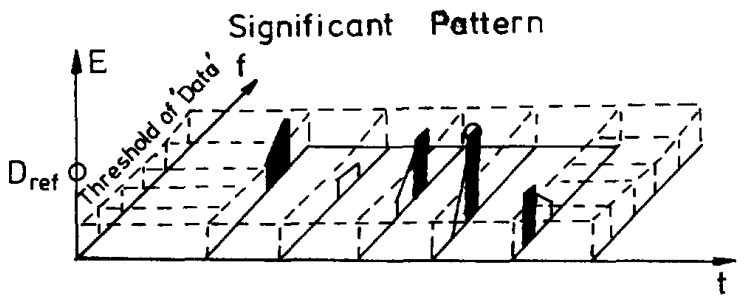

(b)

Fig. 3. (continued)

perception. To model this behavior we must investigate the process of human event detection on single traces once again in more detail.

\section{A. Human Seismogram Inspection}

Initially the seismologist starts his work by getting familiar with the seismogram scalings-assuming that no change will occur in future. He knows by theory how the expected signals should look like, e.g., their average duration, prominent phases, and dominant frequencies. In the routine part, the observer scans the rows of continuous seismogram recordings and will focus on any irregularities which can be higher amplitudes and/or shift of spectral energy over time. Inherent to this process, he permanently tracks the stationary ground noise and estimates its fluctuations. All amplitude variations within these limits are taken as insignificant and will be ignored. When we will interpret detection of earthquakes as image processing, this step will equal the subtraction of stationary background information since only deviations are considered.

After some experience in a given environment, every observer gets familiar with a set of recurrent events-he gets trained to the individual installation. Instead of some general but vague knowledge about earthquake signals, he now can recognize and distinguish the most common seismic events at first glance. Even more, he tries to correlate the oscillations of very weak events that remain visible to the corresponding parts of memorized master events. Driven by an initial event guess, he performs a guided search for those weaker phases which are necessary to confirm his hypothesis. As important as the actual energy spots which can be detected and associated are those master event phases which don't show up in the data because of increased noise background; their predicted absence will also confirm the hypothesis.
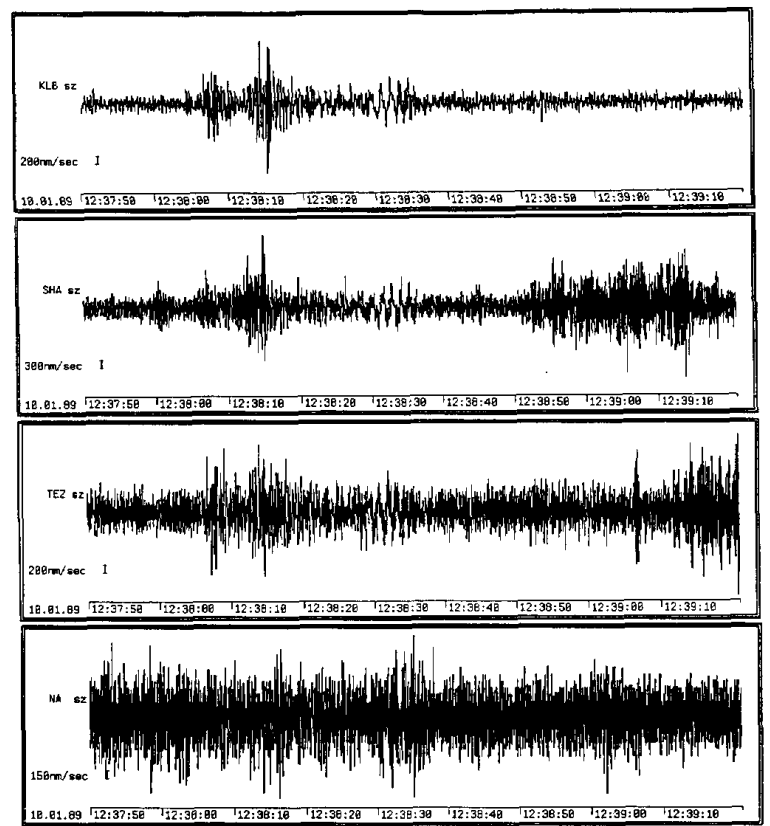

Fig. 4. Four seismograms of a single earthquake taken from the same cluster as in Fig. 1: All seismometer sites form a seismic array and are within $1 \mathrm{~km}$, so the signal energy should be coherent everywhere. The differences in the individual traces are caused by local noise sources. It's some temporary truck traffic on adjacent, badly paved roads for SHA and TEZ; NA is affected by a permanently higher noise level since it is located in the bedstone of the institute building at the university campus.

Overall, this increase in experience will upgrade the detection results.

This whole procedure of human seismogram evaluation shifts from reasoning to unconscious and spontaneous recog- 

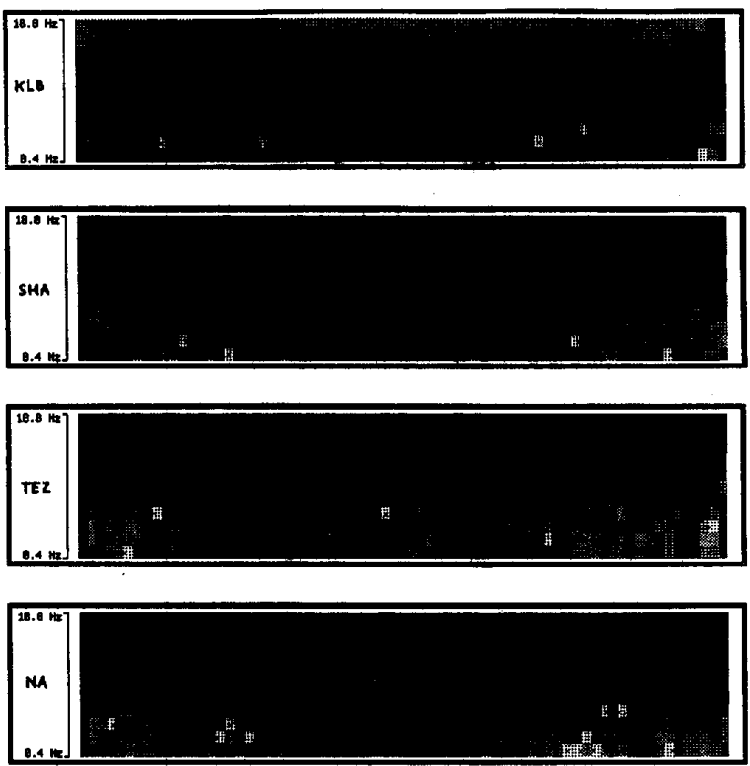

Fig. 5. The corresponding sonograms to Fig. 4 in the same order of stations: The chosen scaling conforms to the log-frequency/log-power transformations commonly used in literature. Although these images are the "true" representatives for sonograms, this scaling yields only poor resolution for earthquake's signal energy.

nition after some weeks or months in routine. It will turn back to the conscious argumentation, however, if any of the seismogram scalings has changed the image, e.g., a new timing resolution has stretched or squeezed the earthquake patterns or some modifications in filter settings and seismometer type have altered the frequency content.

\section{B. Mental Modifications}

The described behavior suggests the interpretation of routine seismogram processing as spontaneous image recognition. As mentioned for the first attempt of sonogram detection, these images can't be the seismograms seen on paper. They are also not the physically interpreted pictures of theoretical reasoning because the spontaneous recognition ignores the differences in the both image axes, i.e., time/amplitude for the seismograms and time/frequency for the sonograms. As a result, we postulate some independent mental images which correspond well to the CRT metaphor of Kosslyn [12]. Comparing the human seismogram evaluation to our first approach of pattern recognition in Fig. 1 we must also state that mental images don't stay unaltered; instead they are dynamical entities that adapt to environmental changes. These modifications occur as image processing before any symbolic interpretation takes place. It could be understood like the correction of illumination effects in photos [20].

For earthquake detection, these modifications perform by (I) sonogram enhancement and (II) pattern adaptation. Both steps can be described by fractional physical models which apply exact theory to the statistical behavior and interaction of signal and noise. For the course of this treaty, the important principles are explained by Fig. 2 and Fig. 3 while the complete algorithm
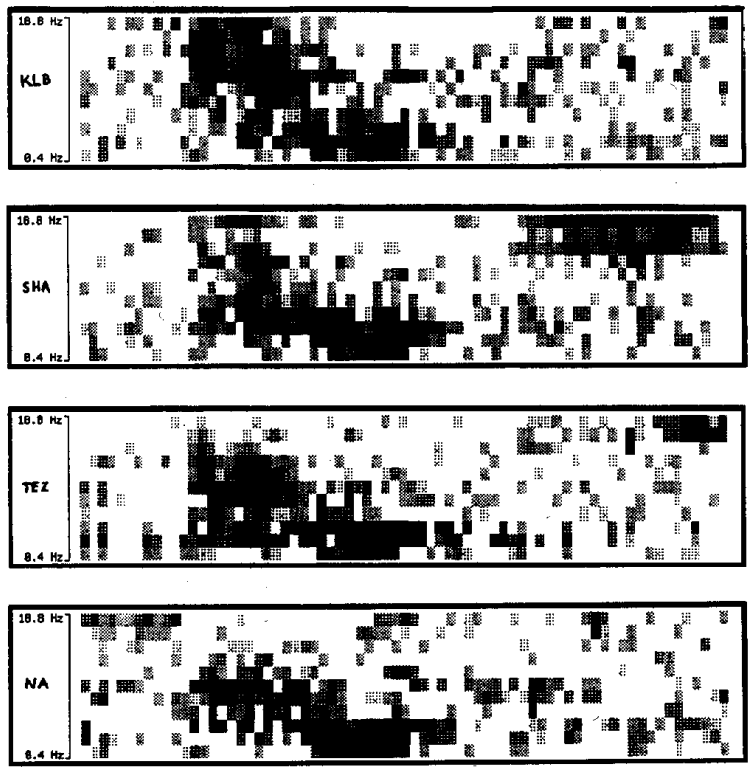

Fig. 6. This time the same information as for Fig. 5 is scaled according to the model of signal to noise interaction in Fig. 2. Now the similarities are evident and allow for simple pattern recognition approaches. The increase in seismic noise at NA, however, masks the upper part of signal energy in the last image. This effect can be predicted and compensated by the pattern adaptation described in Fig. 3. The above images are a good example for the increase in contrast and robustness that can be achieved for mental images by model-based modifications instead of some intuitive heuristics utilized in the initial computation of Fig. 1.

is given in [16]. Our idea of mental image modification is not unique to earthquake processing, for other examples see [21]. The cognitive plausibility of this approach is supported by some known effects in every day vision like the metal rotation [22]. This transformation is also an analytical modification of mental images, the time it takes depends on the number of incremental actions that must be performed to fit both objects. Likewise, the time span for earthquake recognition depends qualitatively on the complexity of image modifications.

Until now, we tried to stress the differences between sonograms either as computer pictures or as mental images. However, there is similarity between them which is not astonishing since both are connected via an isomorphism of second order [23]. The constraints are what makes them different. For the computer sonograms, we expect a constant and comparable mapping of spectral energy without any bias and the best possible resolution. For the mental image, we need the few and only necessary details, the robustness to minor changes, the maximum discrimination on detectable energy and the preservation of amplitude ratios within the signal. The latter constraints are achieved by highly non-linear transformations applied to the computer sonogram, so the mental image corresponds more closely to the impressions recognized by an experienced seismologist than to the visible picture on screen.

\section{Visualized Examples}

By definition, mental images are internal thus invisible. However, we can try to perform similar scalings by computer 


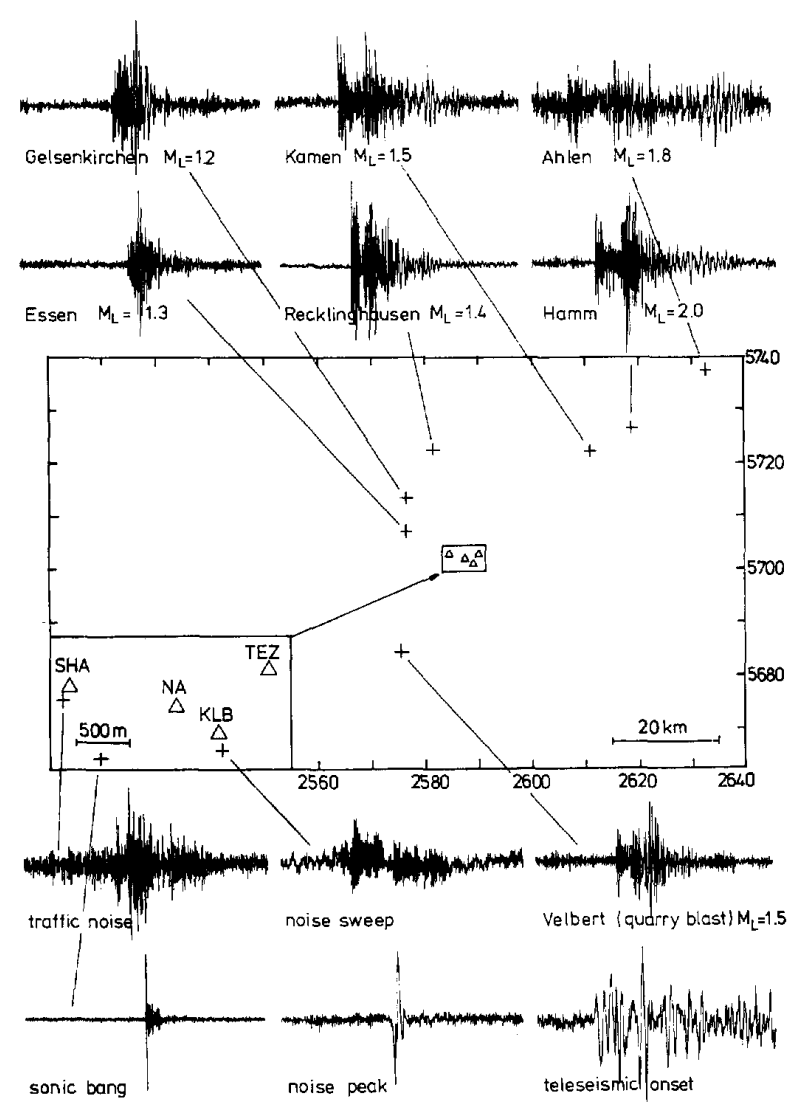

Fig. 7. The approach of pattern adaptation from Fig. 3 allows for some few strong events to define a sufficient pattern set which will guide the recognition of all similar events in a wide range of amplitudes and noise conditions. The input of the 12 displayed events describes most of the local seismicity and the typical noise bursts for the BUG seismic array of Ruhr-University Bochum. No explicit pattern definition as in Fig. 1 is necessary any more; all transformations are performed within the detector system and are transparent to the seismologist.

to compose real images which illustrate the possible impact. Fig. 4 shows the seismograms of one earthquake measured at four different sites of a seismic array, i.e., with comparable signature. So the mental images will be similar to allow for a single pattern to match the situation. In Fig. 5 the common computer sonograms as used in the literature [24] are shown. Although some similarities exist, the differences predominate. After scaling the images to Fig. 2 accordingly, the similarities are obvious in Fig. 6 even for the last trace. Comparing these results to Fig. 1 shows the improvement in image robustness that could be achieved by embedding any image modification into fractional physical models.

Two aspects should be emphasized here: 1) The chosen scaling is highly specialized and only optimum for the detection of transient signal energy in stationary noise. This limitation reminds us that any mental image depends on its question; new investigations will ask for some new images derived from the same set of initial data. 2) We always talk about fractional models that describe part of our situation; any complete theory

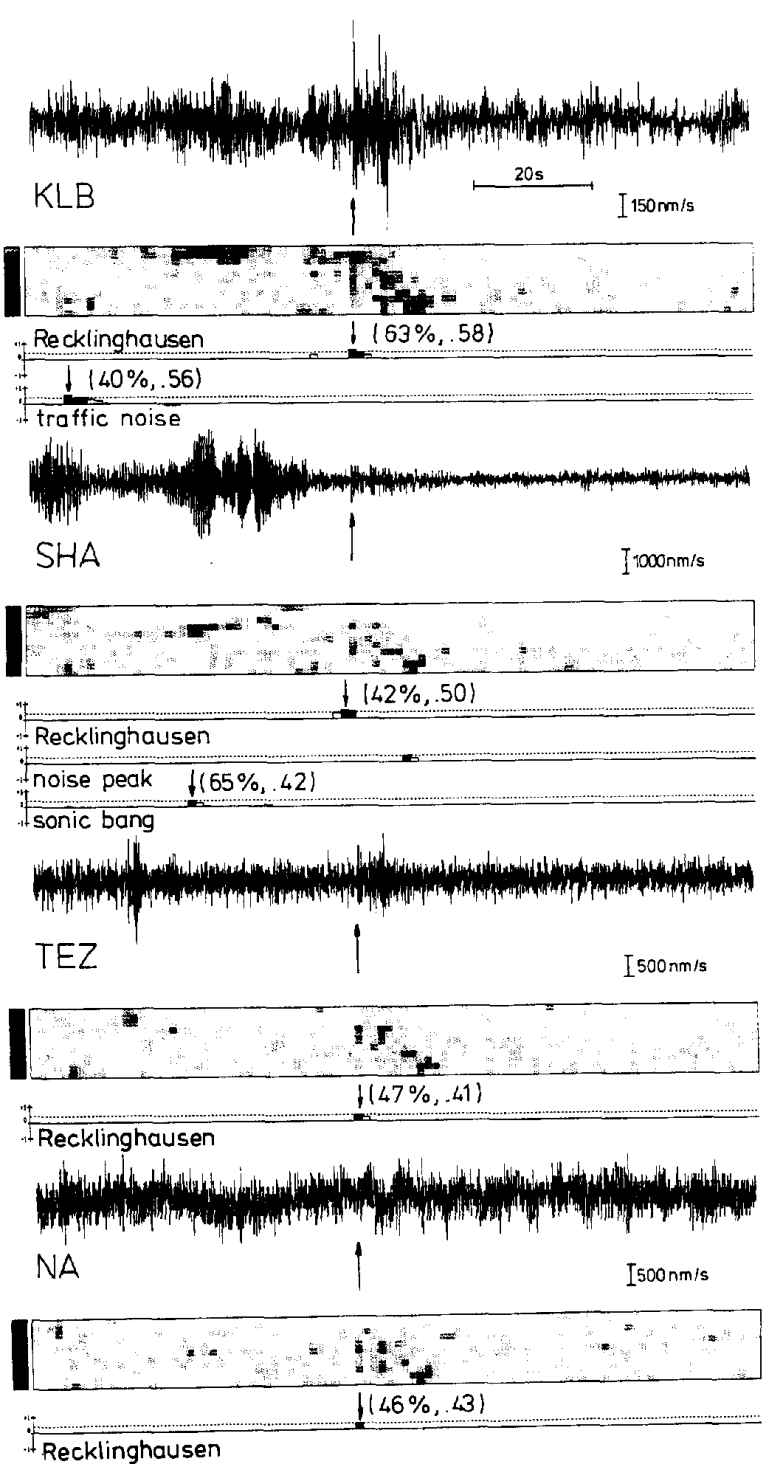

Fig. 8. This example is intended to give an impression for the excellent recognition results. They extend down to extreme noise conditions when detecting a weak event of the Recklinghausen cluster. Although plotted together, the detector decisions were performed independently on all traces. Between seismograms and the possible identification results, we plotted the internally used sonograms for evidence to non-seismologists. Even experienced seismologists will miss the event in the last trace; working with average attention in routine evaluation, he will reliably recognize only KLB.

is still out of reach. We can't predict earthquakes and thus can't utilize matched filters. In this respect we truly deal with artificial intelligence as a technique to handle incomplete knowledge.

Our second fractional approach of pattern adaptation was introduced in Fig. 3 which allows for some few strong events to define the pattern set. This adaptation will guide the recognition of all similar events for a wide range of amplitudes and noise conditions. Additionally, no explicit pattern definition is needed as in Fig. 1 anymore; instead, the input of original 


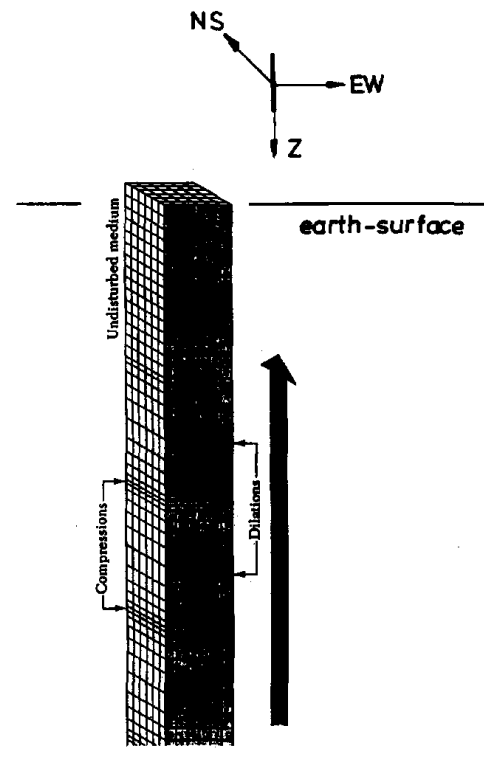

$P$-wave

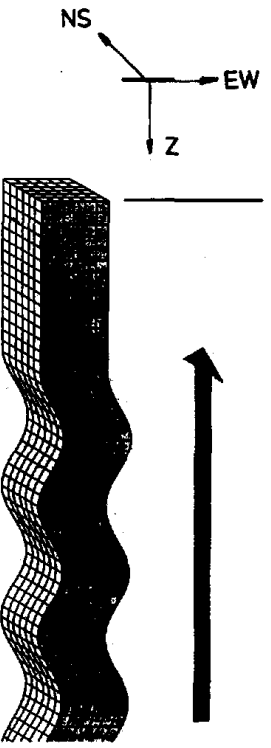

S -wave

Fig. 9. The recognition of S-phase onsets in based on the different propagation properties for these waves compared to the faster $P$ waves. The latter are compressional waves with longitudinal particle motion, so a quite vertically incident earthquake wave shows up on Z-trace. The $S$ or shear wave has particle motion transverse to its spreading, so their registration is dominated by east-west/north-south components.

seismograms-i.e., the unfiltered time series-is sufficient. All the transitions to scaled sonograms appear within the computer system, so they are transparent to the external seismologist even when updating the knowledge base. By this, sonograms are truly mental images not only for a postulated behavior of human scientists but also as realized, internal or invisible images constituting the cornerstone of a knowledge-based system.

To give an example for the functionality of the sonogram detector, Fig. 7 shows the system input of 12 events. They describe the main part of local seismicity and typical noise bursts for the BUG seismic array of Ruhr-University Bochum. Just 12 events made up the complete knowledge base for a one month test run on 740 data segments to yield an average success of $85 \%$. A detailed discussion is given in [16] while Fig. 8 is intended to give an impression of the system's capabilities by detecting a weak event of the Recklinghausen cluster in extreme noise conditions. Although plotted together for the reader's convenience, the detector decisions were performed independently on KLB, SHA, TEZ, and NA. In case of NA, the system even supersedes the results of routine seismogram evaluation by the human seismologist.

\section{MENTAL IMAGES BY DEDUCTION}

The meta-knowledge about mental images gained so far should be sufficient to guide the design of new images. We chose another application in seismology to act as the acid test

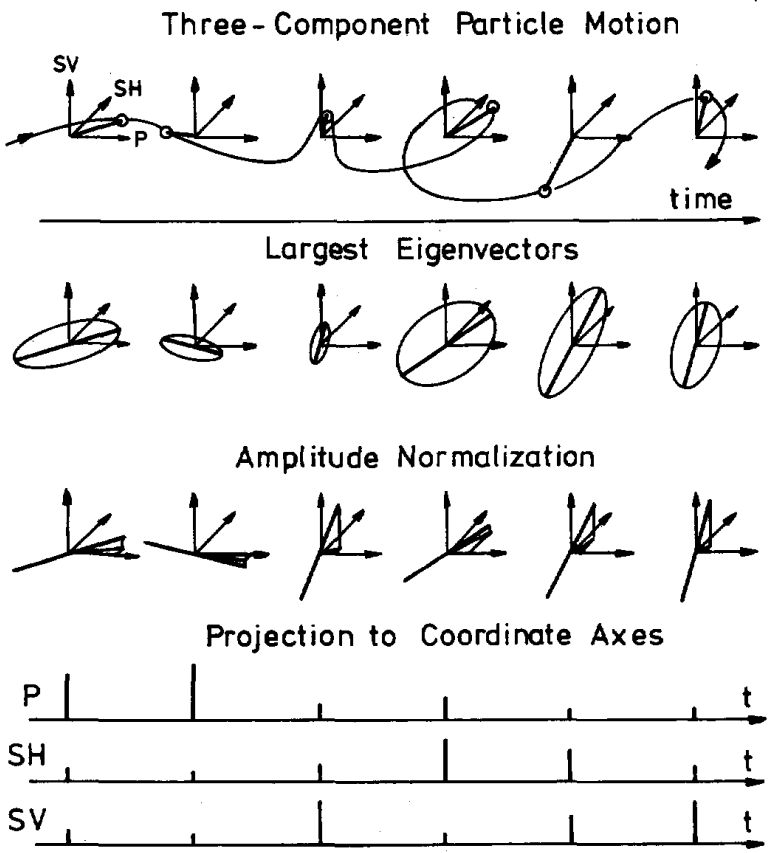

Restriction to Dominant Direction

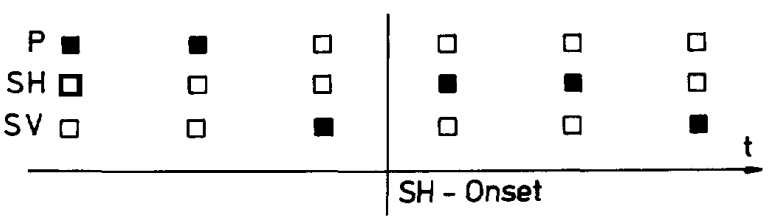

Fig. 10. The automated $\mathrm{S}$ phase determination for local seismicity is complicated by wave field perturbations that are imposed by the fine structure inhomogeneities of upper crust. So the three-component seismograms don't conform to the simple theory of Fig. 9 even when converted from (Z, NS, EW) to $(P, S H, S V)$ coordinate system where $P$ is the direction of incidence. Instead of eigenvectors, we use normalized projections to $(\mathrm{P}, \mathrm{SH}, \mathrm{SV})$ to determine the dominant wave type. The above analysis is performed per frequency band to result in the polarization images of Fig. 11.

since image deduction depends heavily on our domain-specific knowledge to incorporate the necessary physical models and transformations.

One of the most delicate tasks in seismogram evaluation is the determination of $\mathbf{S}$-phase onsets which are necessary to calculate the distance between earthquake hypocenters and seismic stations. While the P-phase onset is just the beginning of the received earthquake seismogram, the $\mathbf{S}$-phase occurs in the middle of the wave train, i.e., in the declining $\mathbf{P}$ coda. Its distinction from the prior oscillations is based on changes in particle motion from longitudinal to transversal-see Fig. 9. However, the sketched propagation of plane waves is very much disturbed in case of high frequency data of local and regional earthquakes. As for the seismogram signature, an exhaustive theory of all encountered effects is still out of reach but seismologists can empirically distinguish $\mathbf{S}$ onsets from $\mathbf{P}$ coda with a very high level of confidence. 


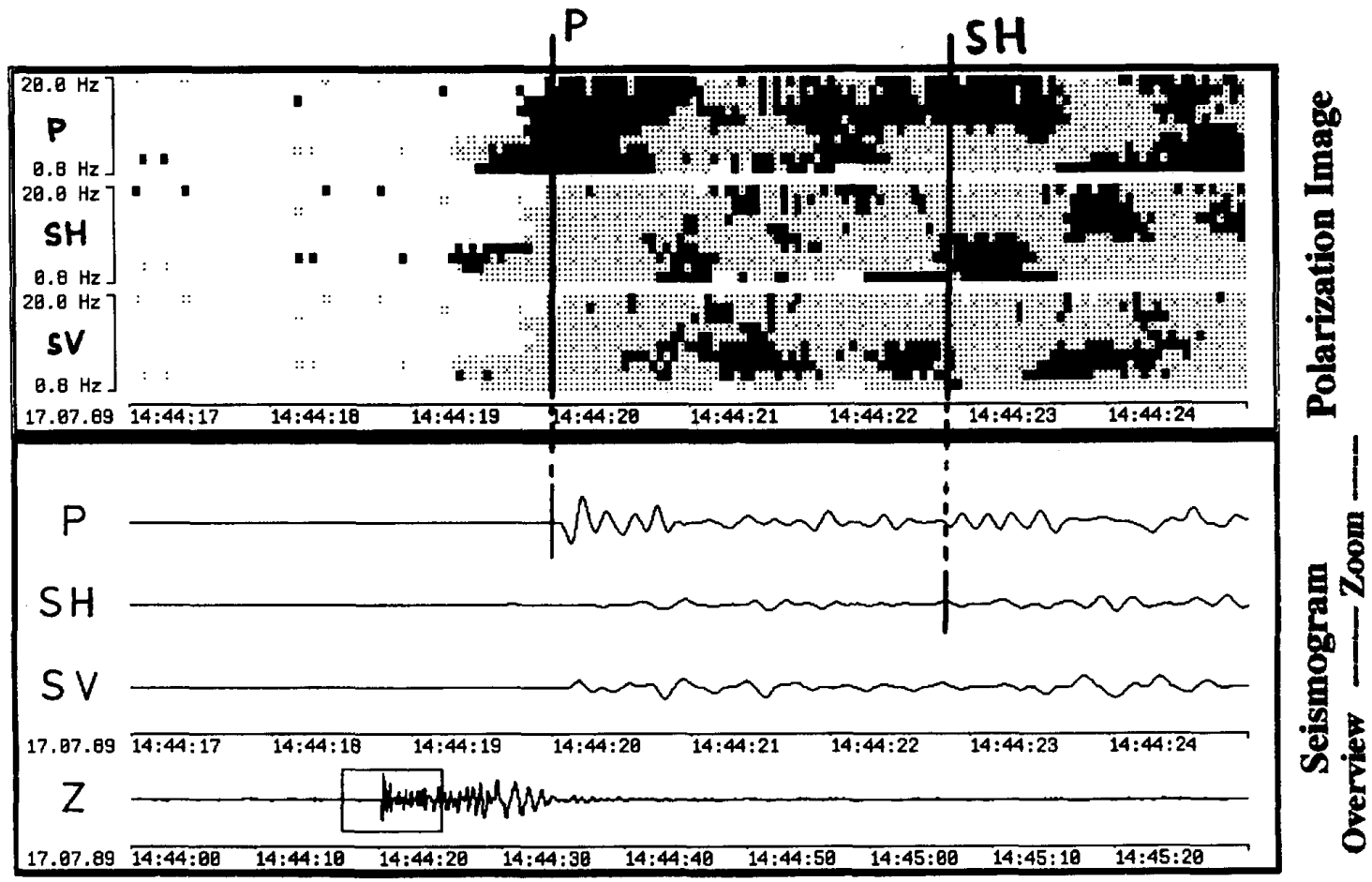

\section{Pattern}

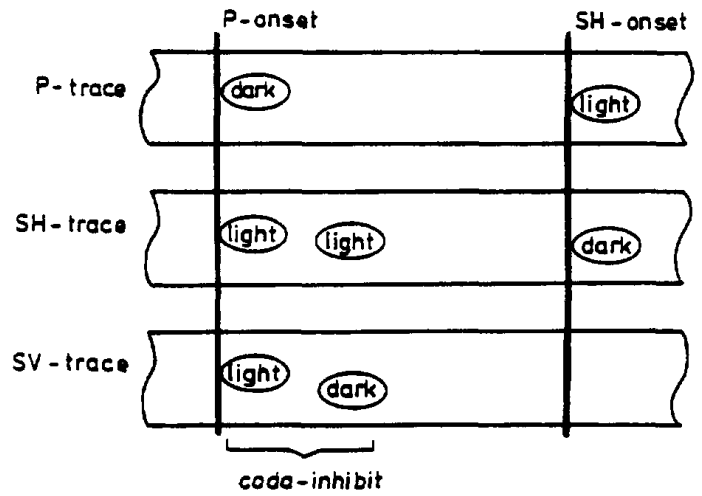

Fig. 11. The composition of suitably scaled polarization images yields robust and simple images to recognize the different wave types. Instead of one pattern per earthquake cluster as for the sonogram detection in Fig. 7, now it is sufficient to define one generic polarization pattern. In case of S waves, it can also resolve the effect of shear-wave splitting by discriminating SH-onsets from SV-precursors (not shown here). Human evaluation on the initial seismograms would have to guess and test for this extremely bad signal to noise ratio.

Many different approaches have been tried to automate the procedure utilizing the simple model of Fig. 9. They started by polarization filtering [25] and reached to the statistical analysis of model conformance [26]. No robust discriminant was found. When we tried to apply the idea of mental images, one extraordinary problem was to deal with the fluctuations of three-component data, i.e., a dependency in four dimensions.
This situation is as hard to visualize as to imagine so no pictorial methods were tested before. With the experience of scaling the sonograms, it took us one year to succeed. We created an interactive analysis tool to visualize the data, we verified the robustness of newly designed polarization images, and implemented a generic polarization pattern. We called it generic because just one pattern is sufficient to 
code all our knowledge. In contrast, the sonogram detection of Fig. 7 demanded one pattern per earthquake cluster. A detailed description of the algorithm, the dependencies on prior knowledge and our test results is given in [17].

Altogether, we could reliably determine S onsets for $67 \%$ of weak local events. Part of the chosen transformations should here be explained by Fig. 10. Commonly the eigenvalue decomposition is used to analyze polarization characteristics which introduces a coordinate system that is aligned to the eigenvectors and thus "flips" in space. Instead, we used projections to some fixed directions which corresponds to shadow casting in the everyday vision. Obviously, this has more cognitive plausibility than sitting on top of a flipping vector which makes us feel dizzy. The other image modifications of Fig. 10 were already introduced for sonograms: amplitude normalization, resolution of frequency dependencies and background blanking. They achieve an image scaling that conforms to the subjective weighting of information by the seismologist-a qualitative confirmation by the dominant characteristics from Fig. 9. In Fig. 11 we see some three-component data with an extremely weak $S$ phase. Still the determined onset times are correct, they are picked by the generic polarization pattern in the polarization image at top.

\section{CONCLUSION}

Pictorial knowledge representation was introduced as an appropriate approach to perform scientific recognition tasks on mass data for further symbolic postprocessing. The experiences gained so far can be summarized in some metaknowledge about the general features of analogue representation forms: They differ from the initial data by lower resolution and limited dynamic range, they focus on changes by background blanking and they can adjust to different environmental conditions by model-based modifications. The optimum transformation is always problem dependent and must be derived from the initial data for every new task that should be performed.

The effects of scaling can be seen as the difference between a photographic picture and the abstracted pen-sketch. The latter is far more robust and thus more suited for the automated pattern recognition. Our computer images should mirror these pen-sketches; so we designed them to correspond best to the subjective weighting of recognized impressions by the scientist. As we follow the ideas of mental images and mental modifications in human cognition, it is possible to address the internal, pictorial representation of knowledge inside the computer system as mental images also.

Pattern recognition on mental images allows for symbolic processing but the patterns themselves stay pictorial and are not part of the image interpretation. Although some modification procedures are based on fractional physical models, the approach of mental images is especially suited to automate the handling of real-life data. Despite their complexity, they can be recognized by the scientist even when they are not fully understood or predictable by the actual theories of natural sciences. In the pure task of pattern recognition, some similarities exist with neural networks; however, they just hide the fundamental difference. For mental images, the input of very few patterns is sufficient to process a wide variety of unknown situations. Neural networks require the opposite relation to get sufficiently be trained. In this way PR on mental images and the concept of mental modifications is a top-down approach like expert systems are, but with an analogue instead of the propositional knowledge representation.

When we started to describe solutions for some specific computer calculation tasks, why did we need the concept of images at all? Are images still mental if they can be visualized? These and other questions may remain unsolved but it was not the scope of this paper to find the final answer. These questions are as complex as our speculations if human thoughts can be captured by sentences or computer clauses. The presented examples should demonstrate, however, that some properties of our knowledge representation like contrast, resolution, and reproducibility are best described by their analogy to images. Also some cognitive plausibility could be demonstrated for the necessary image modifications. And finally the achieved results are superior to any other approach tested on the given seismological applications right now.

\section{REFERENCES}

[1] B. A. Bolt, Inside the Earth. San Francisco, CA: Freeman and Co., 1982.

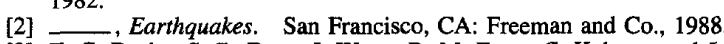

[3] T. C. Bache, S. R. Bratt, J. Wang, R. M. Fung, C. Kobym, and J. W. Given, "The intelligent monitoring system," Bull. Seism. Soc. Am., vol. 80, no. B6, pp. $1833-1851,1990$.

[4] C. Chiaruttini, V. Roberto, and F. Saitta, "Artificial intelligence techniques in seismic signal interpretation," Geophys.J. Int., vol. 98, no. 2, pp. 223-232, 1989.

[5] R. R. Johnson, T. W. Canales, D. L. Lager, C. L. Mason, and R. M. Searfus, "Interpreting signals with an assumption-based truth maintenance system," Proc. Int. Soc. Opt. Eng., vol. 786, pp. 332-337, 1987.

[6] R. M. Searfus, "Using expert systems in treaty verification," in Energy and Technology Review. Livermore, CA: LLNL, Jan. 1989, pp. 26-33.

[7] M. Joswig, "Pattern recognition for earthquake detection," Bull. Seism. Soc. Am., vol. 80, no. 1, pp. 170-186, 1990.

[8] W. H. K. Lee and S. W. Stewart, Principles and Applications of Microearthquake Networks. New York: Academic Press, 1981.

[9] Hsi-Ho Liu and King-Sun Fu, "An application of syntactic pattem recognition to seismic discrimination," IEEE Trans. Geosc. Rem. Sens., vol. GE-21, no. 2, pp. 125-132, 1983.

[10] K. R. Anderson, "Syntactic analysis of seismic waveforms using augmented transition network grammars," Geoexploration, vol. 20, pp. $161-182,1982$.

[11] J. R. Anderson, The Architecture of Cognition. Cambridge MA: Harvard Univ. Press, 1983.

[12] S. M. Kosslyn, Image and mind. Cambridge, MA: Harvard Univ. Press, 1980.

[13] Z. W. Pylyshyn, "The imagery debate: Analogue media versus tacit knowledge," J. Psycholog. Rev., vol. 88, pp. 16-45, 1981.

[14] J. Haugeland, Artificial Intelligence: The Very Idea. Cambridge, MA: MIT Press, 1985.

[15] M. Joswig, "Automated seismogram analysis for the tripartite BUG array: An introduction," Comp. and Geoscien., vol. 19, no. 2, pp. 203-206, 1993.

[16] , "Single-trace detection and array-wide coincidence association of local earthquakes and explosions," Comp. and Geoscien., vol. 19, no. 2, pp. 207-221, 1993.

[17] E. Klumpen and M. Joswig, "Automated reevaluation of local earthquake data by application of generic polarization patterns for $\mathrm{P}$-and S- - onsets," Comp. and Geoscien., vol. 19, no. 2, pp. 223-231, 1993.

[18] M. Joswig, "Chances and limits in automated seismogram processing", in Proc. 23. Gen. Assembly of ESC, V. Schenk and D. Mayer-Rosa, eds. vol. 1, pp. 197-200, Czechoslovak Academy of Sciences, Prague, 1992. 
[19] J. von Neumann, The Computer and the Brain. New Haven: Yale Univ. Press, 1958.

[20] P. H. Winston, Artificial Intelligence. Reading MA: Addison-Wesley, 1984, pp. 361-365.

[21] M. A. Fischler and O. Firschein, Intelligence: The Eye, the Brain and the Computer. Reading, MA: Addison Wesley, 1987, p. 69.

[22] R. N. Shepard and J. Metzler, "Mental rotation of three-dimensional objects," Science, vol. 171, pp. 701-703, 1971.

[23] R. N. Shepard and S. Shipman, "Second-order isomorphism of internal representations: Shapes of state," J. Cogn. Psychology, vol. 1, pp. 1-17, 1970.

[24] J. R. Evans and S. S. Allen, "A teleseismic-specific detection algorithm for single short-period traces," Bull. Seism. Soc. Am., vol. 73, pp. 1173-1186, 1983.

[25] J. Montalbetti and E. R. Kanasewich, "Enhancement of teleseismic body phases with a polarization filter," J Geophys. J. Royal Astr. Soc. , vol. 21, pp. $119-129,1970$.

[26] A. Christoffersson, E. S. Husebye, and S. F. Ingate, "Wavefield decomposition using ML-probabilities in modelling single-site-3-component records", J. Geophys., vol. 93, pp. 197-214, May 1988.

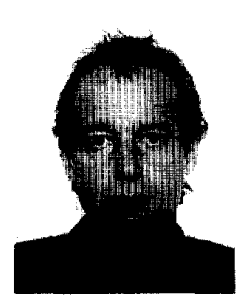

Manfred Joswig (M'91) received the Diplom degree in physics in 1980 and the Doctoral degree in natural science in 1987, both from Ruhr-Universität Bochum, Germany.

$\mathrm{He}$ is currently with Lehrstuhl für Meßtechnik of Technical University Aachen, Germany, working on image processing for medical data (X-ray, ultrasonics). For the last 12 years, he was with Institut für Geophysik, Ruhr-Universität Bochum,

Germany, working on computerized measurement of earthquake data and methods for automated seismogram interpretation. Before he developed a measurement device for acoustical impedances of human eardrums at Lehrstuhl für allgemeine Elektrotechnik und Akustik, Ruhr-Universit ät Bochum. His research interests focus today at image and signal processing, artificial intelligence, cognition and their application to seismology, medical diagnostics and physics.

Dr. Joswig is a member of the German Societies for Physics (DFG) Geophysics (DGG) and Informatik (GI), the American Geophysical Union (AGU), and the Seismological Society of America (SSA). 